\title{
A benchmarked framework for geometric digital twinning of slab and beam-and-slab bridges
}

\section{Ruodan Lu}

Lecturer in Digital Construction, School of Architecture, Building and Civil Engineering, Loughborough University, Loughborough, UK; Senior Member, Darwin College, University of Cambridge, Cambridge, UK (corresponding author: r.lu@|boro.ac.uk)

\section{loannis Brilakis}

Laing O'Rourke Reader, Department of Engineering, University of Cambridge, Cambridge, UK

This paper describes the design, implementation and benchmarking of a framework for automating the process of geometric digital twinning for existing slab and beam-and-slab bridges. Called Lukis, the framework followed a top-down strategy to detect and twin bridge concrete elements in point clouds into an established data format. Existing software packages require modellers to spend many hours generating shapes to fit point-cloud sub-parts. Previous methods can generate surface primitives combined with rule-based classification to produce cuboid and cylinder models. While these methods work well in synthetic datasets or simplified cases, they encounter challenges when dealing with real-world point clouds. This challenge was tackled by investigating the entire workflow of geometric digital twinning for bridges and proposing a new framework to auto-generate bridge objects without needing to generate low-level surface primitives. The framework was implemented on a single software platform. Experiments demonstrated its ability rapidly to twin geometric bridge concrete elements. Compared with manual operation, the framework reduced the overall twinning time by at least $95 \%$ while the twinning quality (spatial accuracy) was improved. Lukis is the first framework of its kind to have achieved geometric digital twinning for primary concrete elements of bridges on one platform. It has laid foundations for researchers to generate semantically enriched digital twins.

\section{Notation}

$\begin{array}{ll}A(x) & \begin{array}{l}\text { area of the cross-section of a slice } \\ a\end{array} \\ b & \text { start of the volume along the } x \text {-axis } \\ \mathrm{I} & \text { end of the volume along the } x \text {-axis } \\ K & \text { identity matrix } \\ n & \text { number of slices with equal thickness } \\ P_{\mathrm{r}} & \text { precision } \\ p\left(x_{\text {pix }}, y_{p i x}\right) & \text { screen coordinates } \\ p\left(x_{\mathrm{w}}, y_{\mathrm{w}}, Z_{\mathrm{w}}\right) & \text { world coordinates } \\ R & \text { recall } \\ \mathrm{R}, \boldsymbol{T} & \text { extrinsic parameters of a camera (rotation matrix } \\ & \text { and translation vector) } \\ V & \text { volume of an arbitrary three-dimensional solid } \\ Z_{\mathrm{c}} & \text { world coordinates in the camera system } \\ \Delta x & \text { slice thickness; } \Delta x=(b-a) / n\end{array}$

\section{Introduction}

Bridges are fundamental to a nation's transport infrastructure network. Every time that a bridge collapse occurs, the public is reawakened to the fact that its most iconic structures need constant vigilance in the form of maintenance. In the USA and UK, federal and state agencies and highway authorities have developed bridge inspection and rating tools (FHWA, 2012; Flaig and Lark, 2000) aimed at prioritising bridge rehabilitation projects.

The USA and the UK spend US\$12.8 billion and $£ 4$ billion every year, respectively, to address deteriorating bridges and maintain their road network (ASCE, 2013; NAO, 2018). The reasons behind these enormous costs are mainly because the manual inspection data available in existing bridge-management systems (BMSs) do not meet the standard of information needed for sound decision making (ASCE, 2017). Innovative digital bridge documentation techniques are urgently needed to boost bridge-management productivity.

Building information modelling (BIM) has been introduced and adopted for many decades in the architecture, engineering and construction sectors (Sacks et al., 2018a). However, BIM is not an appropriate model for real-time operational response. Its designcentric and static nature impedes its usage within the operation and maintenance $(\mathrm{O} \& \mathrm{M})$ phase and life-cycle asset management. Statistics show that the percentage of using BIM technology on over half of the infrastructure projects in the USA and Europe has doubled or even tripled from 2013 to 2017 (Buckley and Logan, 2017). However, the implementation concentrates in the design stage and is almost absent in the O\&M phase (Table 1).

A digital twin (DT) (Grieves and Vickers, 2016; Parrott and Lane, 2017) comprises both the three-dimensional (3D) geometry of the infrastructure components and a comprehensive set of semantic information, including material, functions and relationships between the components (Borrmann et al., 2018a). It could be further enriched with other information, similar to what researchers implement in BIM, such as sensor data (Davila Delgado et al., 2017) and damage information (Hüthwohl et al., 2018). This is particularly useful for bridge inspection practice that is currently based on 
Table 1. Project stage at which BIM technology provides value (Buckley and Logan, 2017)

\begin{tabular}{|c|c|c|c|c|}
\hline & USA: \% & UK: \% & France: $\%$ & Germany: \% \\
\hline \multicolumn{5}{|l|}{ Before design begins } \\
\hline Preplanning (USA)/brief (UK, France, Germany) & 7 & 0 & 4 & 2 \\
\hline Predesign (USA)/concept (UK, France, Germany) & 15 & 22 & 10 & 19 \\
\hline \multicolumn{5}{|l|}{ During design } \\
\hline Design development (USA)/developed design (UK, France, Germany) & 36 & 49 & 49 & 44 \\
\hline Construction documentation (USA only) & 11 & - & - & - \\
\hline \multicolumn{5}{|l|}{ Bidding/construction/installation } \\
\hline Bid letting (USA) & 1 & - & - & - \\
\hline Production (UK, France, Germany) & & 13 & 20 & 22 \\
\hline Construction (USA)/installation (UK, France, Germany) & 28 & 7 & 3 & 13 \\
\hline \multicolumn{5}{|l|}{ Post-construction } \\
\hline Project closeout (USA)/as built (UK, France, Germany) & 0 & 7 & 12 & 0 \\
\hline Maintenance (USA)/use (UK, France, Germany) & 0 & 2 & 1 & 0 \\
\hline
\end{tabular}

Numbers in bold to highlight the 'maintenance' figures

qualitative visual assessment and high-level comparisons, as the texture and damage data can be properly integrated with the geometry at the element level of the virtual model of a bridge.

The essential feature of DTs is their 3D geometry (Borrmann and Berkhahn, 2018) so that 'geometric' is used to highlight the DT with only its geometry data (gDT). Such gDTs can be generated using the point clouds collected with laser scanners. Laser scanning is a technique for capturing the exact size and shape of an object into the computer world as a collection of $X Y Z$ coordinates. The following sections introduce the exchange information requirements (EIRs) of DTs and then provide a brief review of software solutions to see how far they have achieved in terms of the degree of automation on twinning from point clouds, according to the EIRs.

\subsection{Exchange information requirements}

The end users of bridge DTs include but are not limited to inspectors, structural engineers and decision makers. The EIRs (ISO, 2017) define the information that will be required by the end users from both their own internal team and suppliers/subcontractors for the development of the project and for the operation of the completed built bridge. The EIRs should clearly articulate the information requirements for each supplier/subcontractor and describe the expected information deliverables in terms of documents, model files and structured information. However, the nature of the EIRs depends on the complexity of the project and the experience and the requirements of the end users (Ashworth et al., 2019).

Experienced end users may develop very detailed EIRs, while others may only set out high-level requirements and some basic rules, leaving the supplier/subcontractors to propose how those requirements will be met (Ashworth et al., 2017; Hafeez et al., 2016). Detailed EIRs of a DT are extensive and complex. Developing such thorough EIRs is outside the scope of this study. Given the dynamic and real-time updated nature of a DT, the following bullets outline the fundamental information that a bridge DT should contain for a specific moment. Broadly, EIRs should include the following.

- EIR 1: component-level digital representation. This means that the geometric resolution of a bridge DT should be at the component level, not the assembly level or even a lower level (Sacks et al., 2017). Bridge component types include primary (e.g. pier, girder, pier cap and deck) and secondary (e.g. wing walls and handrails) elements (Kedar, 2016). Components that are totally occluded or that are too small or invisible to be discerned due to insufficient scan resolution are not provided.

- EIR 2: component explicit geometry representation and property sets. This means that a bridge DT is prepared for the use as an 'inspection digital model' in a BMS. A bridge DT should represent the as-is geometries of components (Borrmann and Berkhahn, 2018). Existing methods of documenting bridge data measure geometries accurate to 10 or $5 \mathrm{~cm}$ and round them up (Kedar, 2014). This is a rough estimation and notation. Defining the level of geometric detail (geometric spatial accuracy) of a bridge DT is beyond the scope of this research.

- EIR 3: component taxonomy. The components making up a bridge DT should not only be modelled but also be identified and labelled with their component types (Koch and König, 2018; Ma et al., 2018).

- EIR 4: component implicit information such as spatial semantics of attributes and structural relationships, material, cost and schedule (Fink, 2018; Teizer and Melzner, 2018; Treeck et al., 2018). A bridge DT should be sufficiently semantically meaningful to provide most of the information needed for decision making concerning the repair, retrofit or build of a bridge (Sacks et al., 2018b).

- EIR 5: component damage information and the association with the bridge parts. Damage type (e.g. structural/nonstructural crack, spalling, scaling and efflorescence), location and orientation should be exactly identified and embedded 
into the bridge DT along with the texture/image data for each visible component (Hüthwohl et al., 2018).

A bridge DT should also be exchanged between various project participants who use different platforms. Therefore, EIRs should also include the following

- EIR 6. All information requirements should be presented in a vendor-neutral data format, such as Industry Foundation Classes (IFC) (Borrmann et al., 2018a; Koch and König, 2018; Sacks et al., 2018a).

There are already many capable software packages on the market, such as Autodesk, Bentley, Trimble and ClearEdge3D, that provide the most advanced digital twinning solutions. For example, ClearEdge3D can automatically extract pipes in a plant point cloud as well as specific standard shapes such as valves and flanges from industry catalogues followed by fitting built-in models to them through a few clicks and manual adjustments (ClearEdge3D, 2019). This means that ClearEdge3D can achieve a certain degree of automation, as EIRs 1, 2 and/or 3 can be partially automated. However, the spec-driven component library of ClearEdge3D can only recognise and fit point-cloud sub-parts with standardised shapes based on an industry specification table.

Limitations of state-of-the-art modelling software application have been highlighted in research (Agapaki et al., 2018). Modellers must first manually segment a point cloud into sub-parts and then manually fit 3D shapes to them (EIR 1 and EIR 2). Fitting accurate 3D shapes to the segmented point clusters is challenging because the set of allowable primitives is limited in most software applications (Wang et al., 2015) so that modellers must customise unusual shapes. Then, modellers need to enrich the resulting gDT with other explicit and implicit information, such as component taxonomy (EIR 3); connectivity, aggregation and so on (EIR 4); and defects (EIR 5). Then, all EIRs need to be exported or converted in IFC format (EIR 6).

Real-world reinforced concrete (RC) bridge components contain skews and imperfections and cannot be simply fitted using regular generic shapes. This study investigated the entire manual twinning process for existing RC bridges from point clouds using CloudCompare and Autodesk Revit. Up until the end of the process, only EIRs 1, 2, 3 and 6 were partially satisfied. Although the Revit Family Editor provides a powerful capability to create shapes in a free-form manner, almost all the total twinning time is spent on customising shapes and fitting them to corresponding point clusters (note: the manual operation time and results will be presented in Section 4). The significant 'bottlenecks' of the manual operation using software packages are listed as follows.

- Existing software packages can semi-automatically extract standardised shapes in point clouds. However, they cannot automatically extract non-canonical shapes, which are frequently present in $\mathrm{RC}$ bridges. Manual shape customisation is laborious and time-consuming.

- EIRs 1, 2, 3 and 6 can only be manually achieved. EIRs 4 and 5 are unavailable within existing applications.

- No single software can offer a one-stop gDT generation solution. Modellers need to shuttle intermediate results in different formats back and forth between different software packages during the twinning process, giving rise to the possibility of information loss.

To tackle the aforementioned challenges in practice, the authors propose a twinning framework for existing bridges, aiming to meet EIRs 1, 2, 3 and 6 - that is, the EIRs required to generate a gDT with component semantic labels. EIRs 4 and 5 are beyond the scope of this research. In the next section, existing research methods related to EIRs 1, 2, 3 and 6 are reviewed. The paper then outlines the framework in Section 3 followed by demonstration of the experiments in Section 4. The results are discussed and conclusions are drawn in Section 5.

\section{Background}

Previous research methods of twinning infrastructure using point clouds can be divided into two groups according to the EIRs: (a) object-detection methods (EIRs 1 and 3) and (b) 3D solid model-fitting methods (EIRs 2 and 6). Each is summarised in turn in the following sections.

\subsection{Object detection in point clouds}

Object detection aims to meet EIRs 1 and 3. In this work, 'detection' is defined as the combination of clustering (from points to point clusters, i.e. EIR 1) and classification (labelling the point clusters, i.e. EIR 3). This problem has been intensively studied in research. Most existing clustering methods follow a 'bottom-up' approach, which goes from points to surfaces followed by semantic labelling to derive objects (Dimitrov and Golparvar-Fard, 2015; Zhang et al., 2015). Most existing classification methods follow a 'top-down' approach, which employs knowledge such as relationships and contexts to detect specific instances embedded in point clouds or to infer the semantics of components in a geometric model (Ahmed et al., 2014; Riveiro et al., 2016). This paper both reviews clustering and classification methods and investigates how far they have solved these challenges. Specific limitations are also identified.

\subsubsection{Clustering methods}

Existing clustering works usually take the bottom-up approach to meet EIR 1 (Zhang et al., 2014). As its name implies, the bottomup approach pieces together low-level features such as points to generate higher-level features successively. Higher-level features include but are not limited to surface normals (Macher et al., 2017), meshes (Marton et al., 2009), surface patches (Zhang et al., 2015), non-uniform B-spline surfaces (Nurbs) (Dimitrov et al., 2016) and voxels (Vo et al., 2015). Four main methods arise from the literature: random sample consensus (Ransac) (Zhang et al., 2015), region growing (RG) (Walsh et al., 2013), Hough transform (HT) 
(Díaz-Vilariño et al., 2015) and octree paradigm (Xu et al., 2018). While the Ransac algorithm is effective in detecting planar surfaces in the presence of outliers, it suffers from spurious planes, which are frequently produced around the boundaries (Jung et al., 2014). In addition, Ransac requires prior knowledge about the data, meaning that the selection of a fixed number of shape hypotheses implies that a prior estimate of the inlier ratio is available (Schnabel et al., 2007). This is often not the case in the practice of twinning for real-world bridges.

RG is also a widely used scheme for point-cloud clustering, where it has the boundary weakness issue. It excels when the point cloud does not suffer from substantive occlusions. However, it tends to over-segment objects when non-trivial occlusions are present (Dimitrov and Golparvar-Fard, 2015). HT is another commonly used clustering method. Adan and Huber (2011) proposed HT methods to detect walls in building point clouds. HT becomes computationally prohibitive when the number of dimension increases. For example, it requires a five-dimensional parameter space for cylinder detection (Rabbani, 2006). Thus, HT is sensitive to parameter dimensions and cannot be directly applied to shapes characterised by too many parameters. This constraint impedes its use in the detection of bridge objects, which often contain skews and imperfections and cannot be described using generic shapes with limited parameters.

Octree-based (OB) methods have been proposed to tackle the issue of computational complexity (Su et al., 2016). However, the segmentation accuracy of $\mathrm{OB}$ methods is sensitive to the voxel size. Vo et al. (2015) proposed an OB algorithm that can semiautomatically adjust the voxel size using an adaptive octree. However, it faces the difficulty of patch clustering for low-pointdensity regions.

\subsubsection{Classification methods}

Top-down methods are usually used to meet EIR 3, because the aforementioned bottom-up detection schemes are rarely suitable for point-cloud classification (Riveiro et al., 2016). Without highlevel information, it could be difficult to determine whether lowlevel primitives such as local surfaces or voxels belong to the same instance. The top-down approach usually combines a set of engineering criteria and classifies objects in point clouds that meet the criteria. Top-down classification methods are robust because domain-specific information such as known parameters (Ahmed et al., 2014), object instances (Dore and Murphy, 2014) and spatial relationships (Koppula et al., 2011) are invariant to factors such as pose and appearance.

Perez-Gallardo et al. (2017) suggested a semantic model-based system for detecting four object classes in an industrial scene using topological information. Riveiro et al. (2016) used topological constraints to segment a masonry bridge point cloud through normal clustering. However, this algorithm largely depends on data quality so that it is difficult to generalise it to adapt real data suffering from occlusions and non-uniformly distributed points. Ma et al. (2018) leveraged relationship knowledge and shape features to classify bridge 3D solid objects (Figure 1). However, the input of this method needs to be a geometric bridge model (not a point cloud), meaning that it assumes that both EIRs 1 and 2 are already achieved.

\subsubsection{Learning-based methods}

Learning-based methods are seeing a lot of use recently in detecting objects in point clouds, aiming to satisfy both EIRs 1 and 3 at once (Xiong et al., 2013). Maturana and Scherer (2015) proposed a supervised 3D convolutional neural network called VoxNet to classify objects using voxelised data. Instead of transforming a point cloud into 3D voxel grids, Qi et al. (2016) introduced a deep neural network called PointNet, which can directly consume points. These methods often require a substantial down-sampling procedure before they can be fed even in high-performance computing systems such as TensorFlow. However, the resulting compressed datasets often lose feature information along the way.

\subsection{Model fitting to point clusters \\ 2.2.1 Fitting methods}

Model fitting aims to meet EIR 2 in the context of twinning for infrastructure. In other words, model fitting aims to use computer
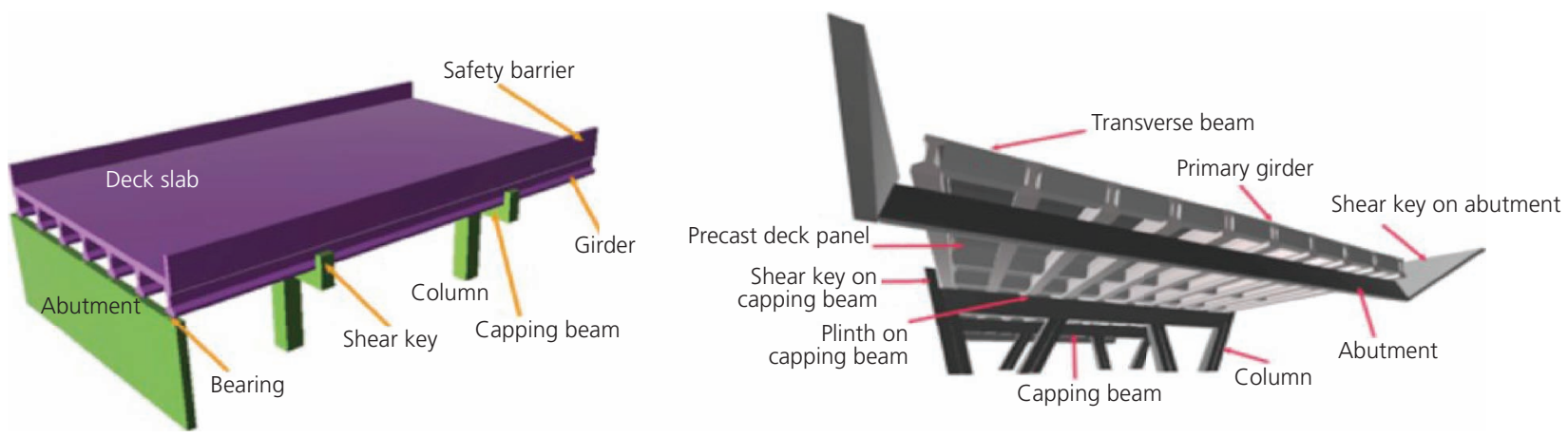

Figure 1. Bridge object classification (EIR 3) (adapted from Ma et al., 2018) 
graphic techniques to form the 3D shape of a point cluster. Existing shape representation methods are categorised into four groups: implicit representation (Schnabel et al., 2007), boundary representation (B-Rep) (Oesau et al., 2014), constructive solid geometry (CSG) (Xiao and Furukawa, 2014) and swept solid representation (SSR) (Ochmann et al., 2016).

Implicit representation is of limited usefulness when twinning real-world bridge components because only a very limited number of shapes can be represented by algebraic formulations. Although B-Rep is the most popular representation in computer graphics, the presence of significant occlusions in point clouds challenges the formation of closed mesh models. CSG methods have been proposed where only elementary primitives such as cuboids and cylinders are used to constitute the built environment.

For example, Zhang et al. (2014) designed a classifier to classify infrastructure components (EIR 3) and fit them with 3D shapes (EIRs 1 and 2). However, it used only cuboids and cylinders to fit piers, girders and decks in synthetic bridge point clouds (Figure 2). Laefer and Truong-Hong (2017) used an SSR method to extrude the cross-sections of steel beams in point clouds, assuming that the standardised profiles do not have any damages or deformation. Table 2 summarises the most related research methods of the gDT generation from point clouds in terms of the application area and the IFC maturity.

\subsubsection{IFC geometric representations}

A gDT made up of multiple fitted geometric shapes needs to be represented in IFC format so that it can be exchanged and adopted by various software tools (EIR 6) (Venugopal et al., 2012). IFC geometry representations can be grouped into four classes (Borrmann et al., 2018b): bounding boxes, curves, surface models and solid models. More specifically, bounding boxes are usually used as placeholders for digital twinning. They can be represented using IfcBoundingBox. IfcCurve and its subclasses can be used to model line objects.
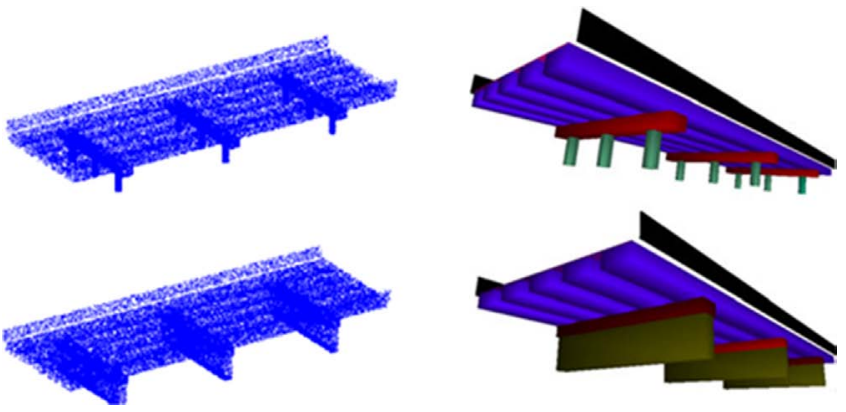

Figure 2. Fitted cuboids and cylinders to synthetic bridge point clusters (EIR 2) (Zhang et al., 2014)

Table 2. State of research on gDT generation from point clouds in terms of IFC maturity

\begin{tabular}{|c|c|c|c|c|c|c|c|}
\hline \multirow[t]{5}{*}{ Area } & \multicolumn{2}{|l|}{ Bridge } & $\begin{array}{l}\text { Riveiro et al. } \\
\text { (2016) } \\
\text { Zhang et al. } \\
\text { (2015) }\end{array}$ & Walsh et al. (2013) & $\begin{array}{r}\text { Lu et al. } \\
\text { (2019) }\end{array}$ & $\begin{array}{l}\text { Zhang et al. (2014) } \\
\text { Lu and Brilakis (2019) }\end{array}$ & $\begin{array}{l}\text { Hüthwohl et al. } \\
(2018)^{a} \\
\text { Ma et al. (2018) }\end{array}$ \\
\hline & Building & $\begin{array}{l}\text { Dimitrov et al. } \\
\text { (2016) } \\
\text { Vo et al. (2015) } \\
\text { Jung et al. (2014) } \\
\text { Truong-Hong et al. } \\
\text { (2013) } \\
\text { Marton et al. } \\
\text { (2009) }\end{array}$ & $\begin{array}{l}\text { Xu et al. (2018) } \\
\text { Wang et al. } \\
\text { (2015) } \\
\text { Díaz-Vilariño et al. } \\
\text { (2015) } \\
\text { Xiong et al. } \\
\text { (2013) } \\
\text { Adan and Huber } \\
\text { (2011) } \\
\text { Koppula et al. } \\
\text { (2011) }\end{array}$ & $\begin{array}{l}\text { Dimitrov and } \\
\text { Golparvar-Fard (2015) } \\
\text { Xiao and Furukawa } \\
(2014) \\
\text { Schnabel et al. (2007) }\end{array}$ & $\begin{array}{l}\text { Qi et al. } \\
\text { (2016) } \\
\text { Armeni et al. } \\
(2016) \\
\text { Su et al. } \\
(2016)\end{array}$ & $\begin{array}{l}\text { Macher et al. (2017) } \\
\text { Ochmann et al. (2016) } \\
\text { Valero et al. (2012, } \\
\text { 2016) }\end{array}$ & $\begin{array}{l}\text { Belsky et al. } \\
(2014)^{\mathrm{a}}\end{array}$ \\
\hline & \multirow[t]{2}{*}{ Industry } & & & & $\begin{array}{l}\text { Patil et al. } \\
\text { (2017) } \\
\text { Su et al. } \\
(2016) \\
\text { Son et al. } \\
\text { (2015) } \\
\text { Ahmed et al. } \\
\text { (2014) } \\
\text { Son et al. } \\
\text { (2013) }\end{array}$ & $\begin{array}{l}\text { Laefer and } \\
\text { Truong-Hong (2017) }\end{array}$ & \\
\hline & & Surfaces & Labelled surfaces & Shapes without labels & $\begin{array}{l}\text { Labelled } \\
\text { clusters }\end{array}$ & Labelled 3D model & $\begin{array}{l}\text { Enriched IFC } \\
\text { model }\end{array}$ \\
\hline & $\rightarrow$ & IFC maturity & & & & & \\
\hline
\end{tabular}


Free-form curved edges (i.e. splines) and curved surfaces are required to model complex geometries. IfcTriangulatedFaceSet can be used to represent polygons with an arbitrary number of edges or triangular mesh. IfcBSplineSurface can be used for representing curved surfaces such as Nurbs. Finally, IfcSweptAreaSolid and its subclasses can be used to represent extruded solids. How to choose an IFC geometric representation depends on the specific application scenario for the object being twinned. Whereas software programs need only low-level triangulated mesh to visualise the geometry, gDTs authoring tools usually use high-level B-Rep- or CSG-based objects so that the geometries could be edited. Details of the IFC geometric representation can be found in the book chapter by Borrmann et al. (2018b).

\section{Proposed framework}

\subsection{Overview}

The authors set out to solve the problem of twinning for existing RC bridges while improving their understanding of the specific challenges and the extent to which they had solved the problem. The main objective of this research is to develop a benchmark framework that paves the foundations for future research to build on.

The top-down approach is based on domain knowledge and relies on a symbolic description of the simplified world. Its goal is to use a set of predefined recognition criteria or rules to mimic human intelligence; hence, it suffers from some inherent limitations. Inevitably, a combinatorial explosion of the number of rules occurs due to the complexity of the environment and it is impossible to predict all situations (particularly unknown ones) that will be encountered by an autonomous entity. Yet, the limitations of the top-down approach are irrelevant in the context of slab and beam-and-slab RC bridges. This is because the level of variance of these types of bridges is relatively low compared with that of other real-world objects. Bridge components are distinct 3D solid objects, and their taxonomies for any given context are finite and well defined. Therefore, a novel top-down framework called Lukis is proposed, which exploits bridge engineering knowledge as guidance for directly extracting labelled point clusters corresponding to bridge components and then reconstructing them into IFC objects.

Real-world bridges are neither perfectly straight nor flat. Bridge geometries are defined by horizontal curved alignments, vertical elevations and varying cross-sections. A slicing-based algorithm is proposed to tackle these difficulties. The algorithm is repeatedly used throughout the whole framework until all the components are detected and modelled. The algorithm can deal with the skew complexity and can quickly select a set of candidate locations for target objects. The global topology of a bridge can also be well approximated using multiple slices. The general thrust behind the slice-based representation is Cavalieri's principle (Kern and Bland, 1948), which serves as the theoretical guidance of the Lukis framework. Given an arbitrary 3D solid along the $x$-axis, extending from $x=a$ to $x=b$ (Figure 3), the solid is divided into $n$ equally thick slices and define the usual partition: $\Delta x=(b-a) / n$ and $x_{i}=a+$ $i \Delta x$, for $i=1,2, \ldots, n$, then Cavalieri's principle states that

$$
V=\lim _{n \rightarrow \infty} \sum_{i=1}^{n} A\left(x_{i}\right) \Delta x=\int_{a}^{b} A(x) \mathrm{d} x
$$

where $A(x)$ is the area of the cross-section of a slice.

The framework twins only the four most important and highly detectable primary components of typical RC slab and beam-and-slab bridges: slab, pier, pier cap and girder. It consists of two successive processes (Figure 4): process 1, detection of the four bridge component types in point clouds, aiming to achieve EIRs 1 and 3, and process 2, run-time model fitting to the point clusters using IFC standards, aiming to achieve EIRs 2 and 6. Specifically, the framework starts with a registered point cloud of an RC bridge. Irrelevant points such as vegetation, trees and traffic are manually removed. It then aligns the cropped point cloud using principal component analysis (PCA) such that its centre axis - that is, horizontal alignment - is roughly parallel to the $x$-axis of the global coordinate system. This step outputs a roughly aligned bridge point cloud, as none of the bridges could be positioned exactly parallel to the axes due to their real-world skewed geometry.

Next, the framework proposes a four-step object-detection method (process 1) to detect the four components underlying in a cropped bridge point cloud in the form of labelled point clusters. A manual refinement procedure is then conducted to remove the noise remaining from process 1 . Next, the framework proposes an IFCobject-fitting method (process 2) to fit the refined labelled point clusters with $3 \mathrm{D}$ IFC objects. The output of process $2,-$ that is, the final output of the framework - is an IFC file of a bridge gDT. The novelty of the framework lies in the fact that it integrates processes 1 and 2 into a single framework that provides a semi-automated method to twin four bridge components from point clouds at one go. Each process is outlined in the following sections.

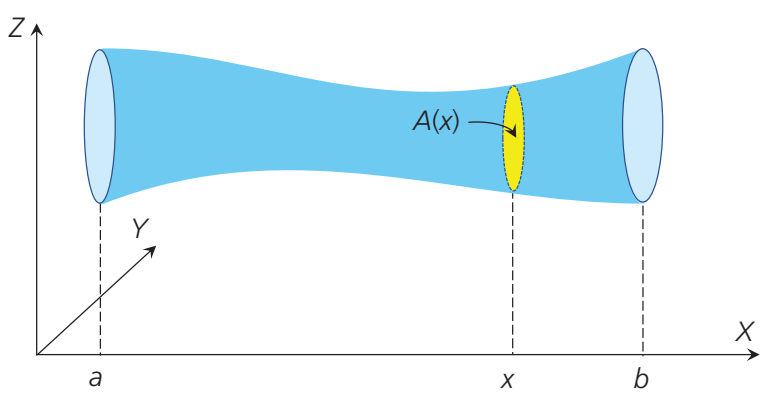

Figure 3. An arbitrary 3D shape represented using its crosssection $A(x)$ along the $x$-axis 


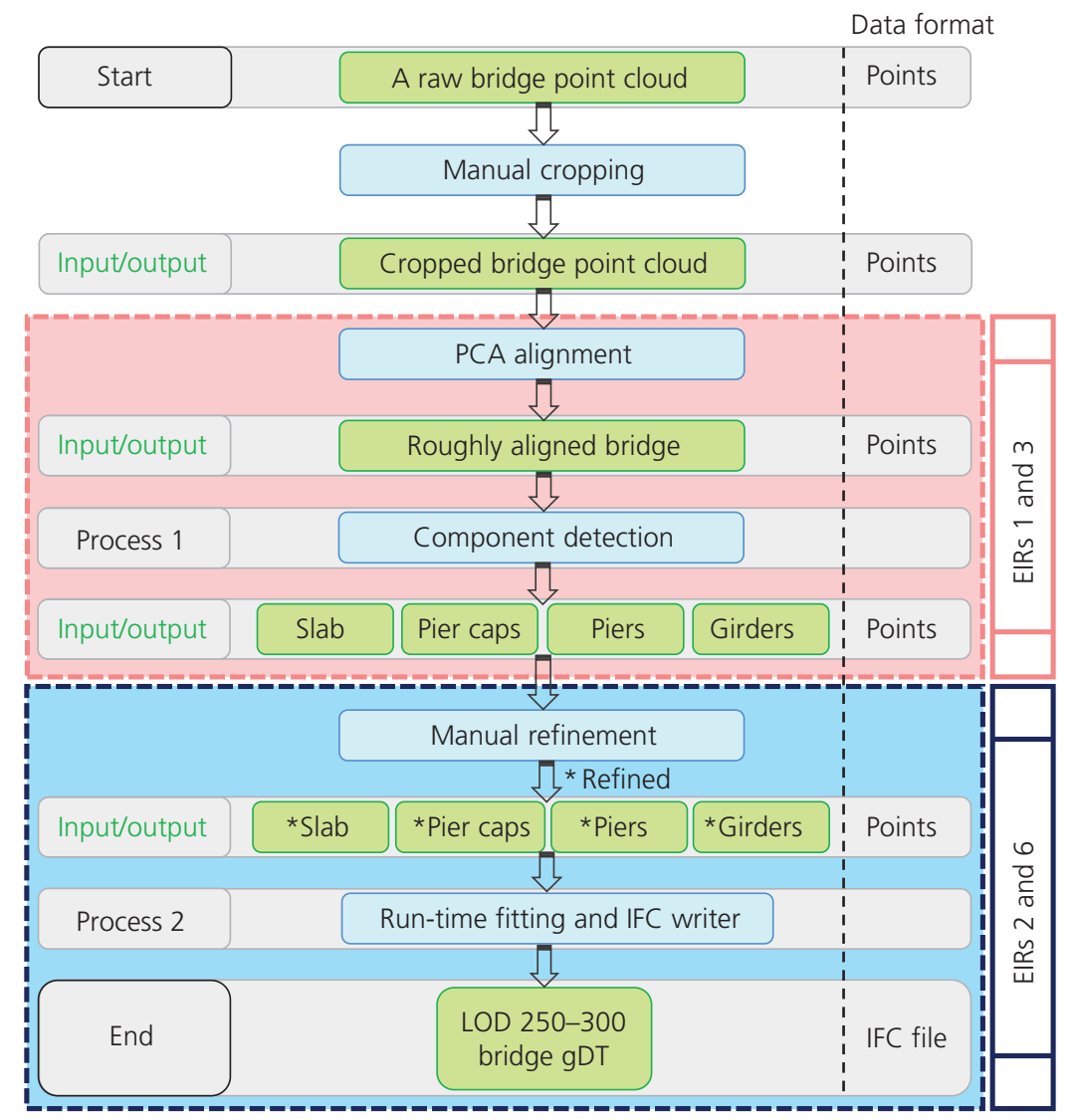

Figure 4. Workflow of the proposed Lukis framework

\subsection{Process 1: primary component detection for EIRs 1 and 3}

The authors used the point cloud of the Nine Wells Bridge to demonstrate the development of process 1 . The bridge was constructed to the south west of Addenbrooke's Hospital in Cambridge, UK, in 2009 to carry a new road over a railway line. It comprises three spans approximately $30 \mathrm{~m}$ long, each constructed from 12 precast concrete 'SY beams' with an in situ concrete deck slab. The bridge also contains a group of four cylindrical piers with a pier cap on top, a wall-like pier and two wall-like vertical abutments as supports. The side view shows its varying elevations over the three spans (Figure 5).

Process 1 bypasses the stage of surface generation altogether and directly obtains labelled point clusters. It breaks down a large bridge point cloud into sub-datasets through a recursive slicing algorithm that is, the method slices the point cloud by means of a 'virtual parallel scalpel' with a specified equal thickness. This algorithm is repeatedly used with sub-datasets until target objects are found and all small detection problems are solved. Step 1 segments a whole aligned bridge point cloud into two classes: pier assembly $a_{m}$ and deck assembly. This is achieved by segmenting the bridge point clouds into multiple slices along the $x$-axis and comparing the height feature of each slice using a discriminative parameter $\rho_{1}$ (Figure 6).
Then, the search area is shrunk to the pier assembly $a_{m}$. Step 2 aims to detect the pier area in pier assembly $a_{m}$ using a discriminative parameter $\rho_{2}$. Each extracted pier assembly $a_{m}$ from step 1 is considered as a miniature of an entire bridge point cloud so that step 2 follows the same strategy as step 1. Next, step 3 further narrows down the search area and detects pier caps in a pier area by investigating the normals. Finally, step 4 aims to detect girders. The entire deck assembly cluster is segmented into several spans followed by using the density histograms to detect girders in each span. All over-segments are merged, and finally, the four labelled point clusters of bridge components (EIRs 1 and 3) are acquired. Details of process 1 and the four-step object-detection method can be found in the paper by Lu et al. (2019).

\subsection{Process 2: IFC object fitting for EIRs 2 and 6}

Process 2 suggests a novel object-fitting method that can twin an $\mathrm{RC}$ bridge into IFC format using the four types of point cluster. The inputs of process 2 are the point clusters generated from process 1 . The output is an IFC file corresponding to the level of detail (LOD) 250-300. The four types of component are represented with detailed geometries through multiple slice models. SSR is preferred wherever possible if the cross-section of each slice is deemed constant. Process 2 consists of two major steps: step 1, geometric feature extraction of point clusters, and step 2, IfcObjects 


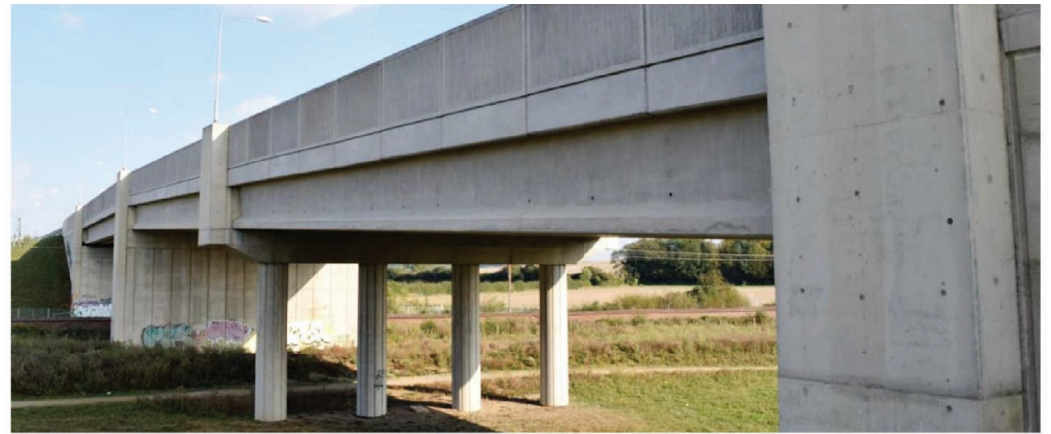

(a)

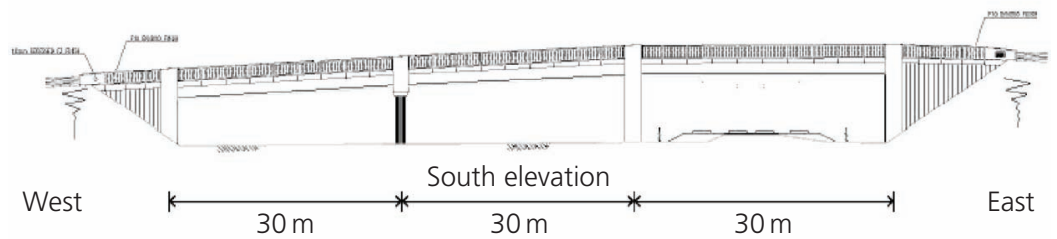

(b)

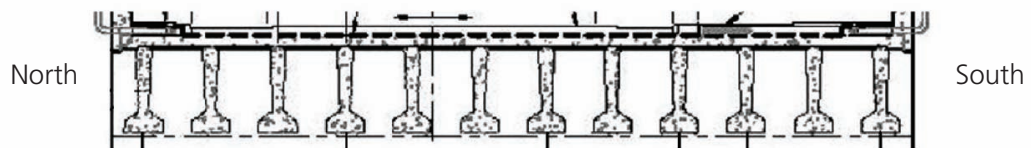

(c)

Figure 5. (a) North-west view, (b) side view and (c) SY beams of the Nine Wells Bridge, Cambridge, UK (Webb, 2014)

fitting to the extracted features. The model view definition proposed by Sacks et al. (2018b) is used, which provides a binding to the IFC4 Add2 standard for exchanging bridge DTs and defines the mandatory IFC entities in a bridge inspection scenario.

For a slab point cluster IfcSlab, a slicing scheme is used that is similar but not identical to that proposed in process 1 to slice the deck slab into multiple slices. The slicing does not take a parallel pattern but is rather oriented along the normal direction of the curved alignment of the slab (Figure 7(a)). The problem of

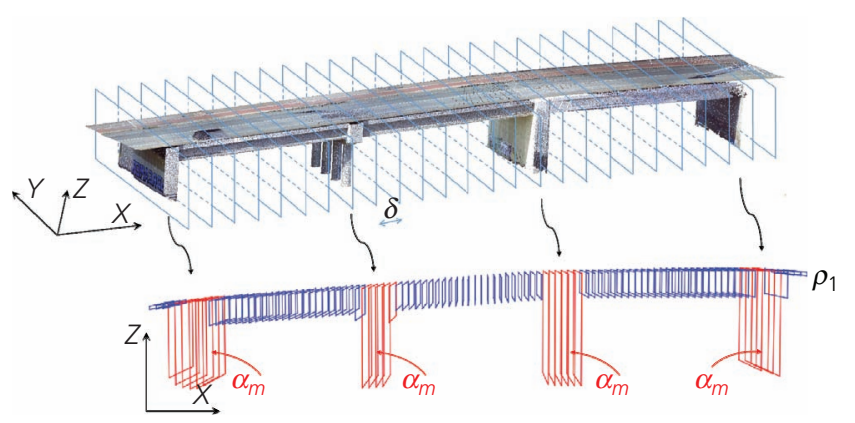

Figure 6 . Slicing along the $x$-axis in step 1 of process 1 twinning the entire slab is transformed into twinning slab slices by assuming that each one of them is straight along its tangent direction and the cross-section of each slice is constant. That is to say, the horizontal alignment can be approximated by concatenating many alignment segments.

Two-dimensional (2D) profiles IfcArbitraryClosedProfileDef and IfcExtrudedAreaSolid were used to describe the cross-section of each extruded slice, which is defined by a $2 \mathrm{D}$ ConvexHull $\alpha$ shape. Pier cap point clusters IfcBeam are also represented as a swept solid using the outline of the $\alpha$-shape on the $X Y$-plane. Then, for pier point clusters IfcColumn, a fuzzy-logic algorithm was used first to classify the cross-section shape into circular, quadrilateral and other shapes. A cylindrical pier was represented as a swept solid using 2D IfcCircleProfileDef, while a quadrilateral pier and piers of other shapes were represented using multiple slice models through IfcArbitraryClosedProfileDef and IfcExtrudedAreaSolid (Figure 7(b)). Finally, for girder point clusters IfcBeam, a template-matching method was used to find the best-match girder type in the existing beam catalogue. Finally, a bridge gDT was acquired in IFC format, made up of four types of component (EIRs 2 and 6). Details of process 2 and the proposed fitting method can be found in the paper by $\mathrm{Lu}$ and Brilakis (2019). 
Smart Infrastructure and Construction Volume 172 Issue 1
A benchmarked framework for geometric digital twinning of slab and beam-and-

slab bridges

Lu and Brilakis

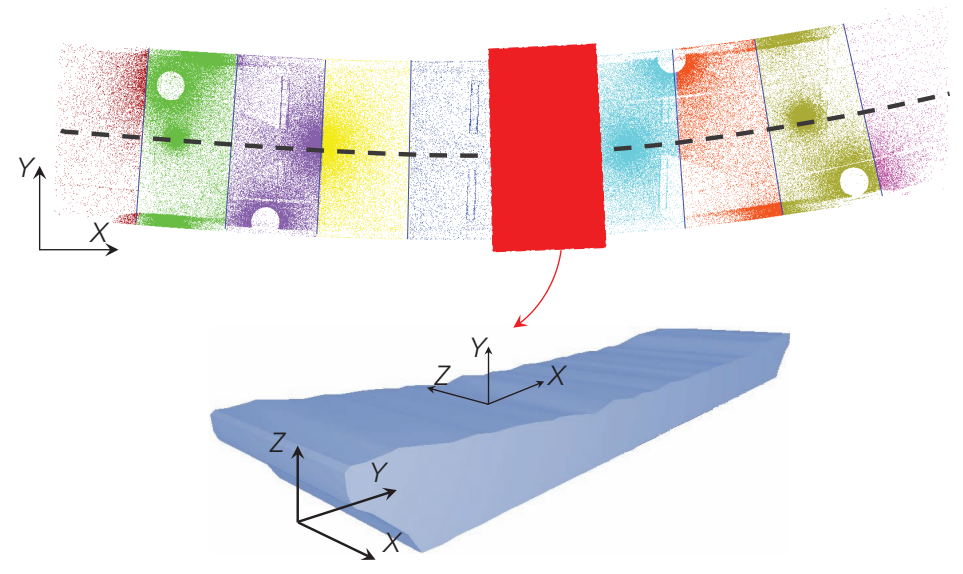

(a)

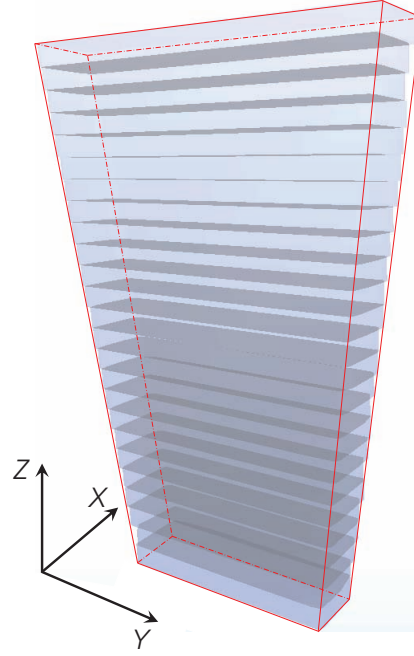

(b)

Figure 7. Slicing and twinning of process 2 for the (a) slab and (b) pier

\section{Research activities}

4.1 Implementation

Ten highway RC slab and beam-and-slab bridge point clouds were used to test the proposed framework (Figure 8). Data can be downloaded from the Zenodo repository of Lu et al. (2018). Detailed statistics of the data can be found in the paper by Lu et al. (2019).
The framework was implemented on Gygax (Hüthwohl et al., 2017) into a proof-of-concept prototype software package on a desktop computer with the following system configurations: central processing unit, Intel Core i7-4790K $4.00 \mathrm{GHz}$; memory, $32 \mathrm{~GB}$; and solid-state drive, $500 \mathrm{~GB}$. Gygax contains three projects: GygaxCore (C\#), GygaxVisu (C\#) and PclWrapper

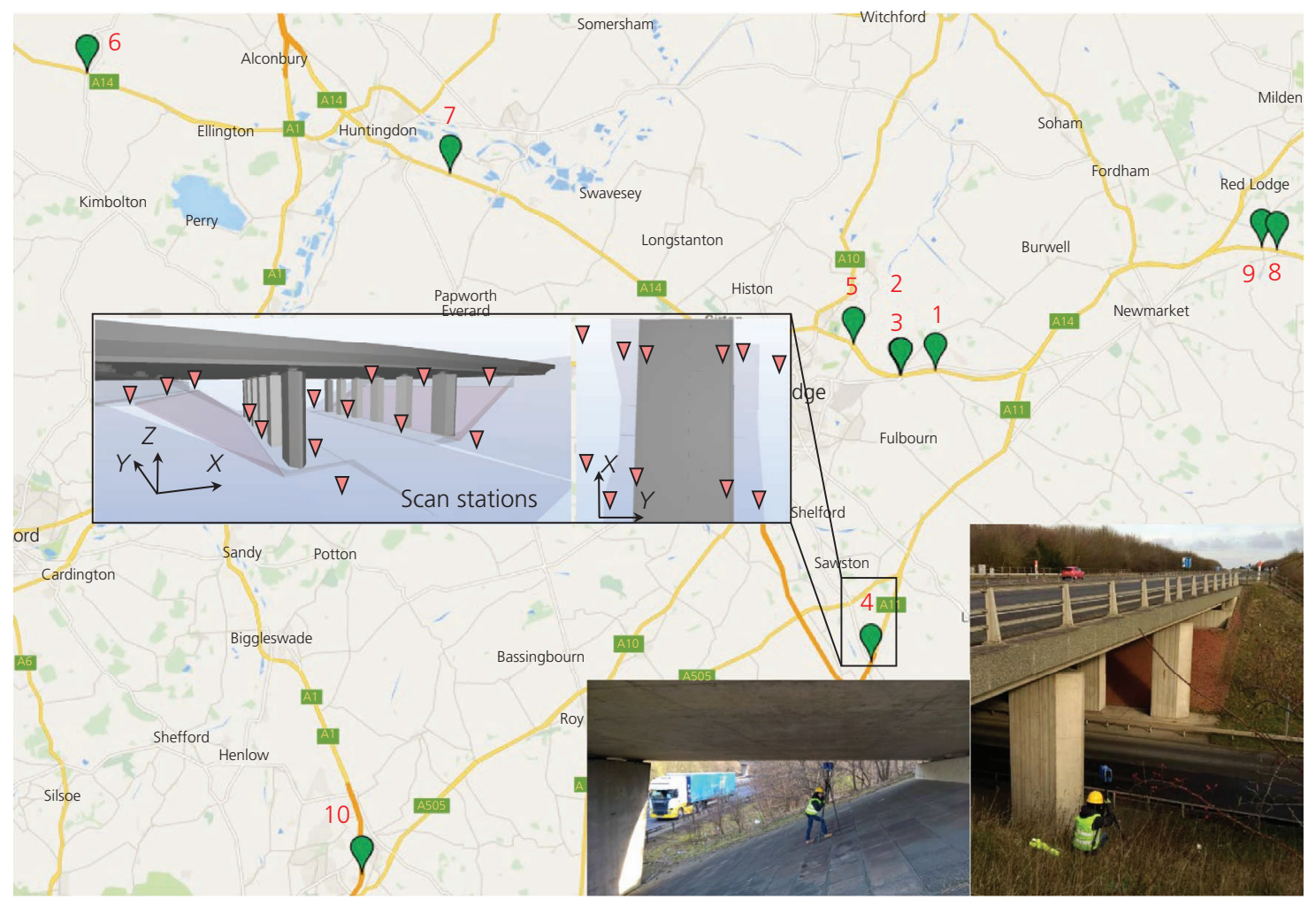

Figure 8. Diagram of locations of the ten bridges, scan stations and on-site activities 
$(\mathrm{C}++)$. GygaxCore defines basic functionalities such as the data structures and interfaces of this platform. GygaxVisu supports visualisation. Helix Toolkit was used to visualise all supported data sources in a single 3D space. PclWrapper allows Gygax to access Point Cloud Library application programming interface in a C\# environment. IFC Engine DLL was used to read and visualise IFC files on a logical and geometrical level.

The raw bridge point clouds were extremely noisy because data were collected with live traffic. Data clean-up is needed before running any experiments. A user-defined 2D clipping polygon function on Gygax was developed to remove manually irrelevant points such as the on-site traffic, vegetation and ground surface (Figure 9). To do so, the current graphics view of $3 \mathrm{D}$ points was mapped onto a $2 \mathrm{D}$ screen by controlling the viewport to conduct the clipping. The $3 \mathrm{D}$ points in the world coordinates $\left(x_{\mathrm{w}}, y_{\mathrm{w}}, z_{\mathrm{w}}\right)$ were transformed into a camera space followed by perspective projection and affine transformation to convert the camera coordinates into the screen coordinates $\left(x_{\text {pix }}\right.$, $y_{\text {pix }}$ ) using

$$
p\left(x_{\text {pix }}, y_{\text {pix }}\right)=\frac{1}{Z_{\mathrm{c}}} K\left[\begin{array}{ll}
\mathbf{I} & 0
\end{array}\right][\mathbf{R} \mid \boldsymbol{T}]\left[\begin{array}{c}
x_{\mathrm{w}} \\
y_{\mathrm{w}} \\
z_{\mathrm{w}} \\
1
\end{array}\right]
$$

where $Z_{\mathrm{c}}$ is the world coordinate $z_{\mathrm{w}}$ in the camera system; $K$ is the intrinsic parameter of a camera; and $\mathbf{R}$ and $\boldsymbol{T}$ are the extrinsic parameters. PCA was then used to align the cropped bridge point cloud followed by implementing process 1 , bridge component detection, and process 2, IFC object fitting, on Gygax as two successive modules. Details of process 1 can be found in the paper by Lu et al. (2019), and details of process 2 can be found in the paper by Lu and Brilakis (2019).

\subsection{Manual operation}

For process 1, three ground-truth (GT) datasets, GT A, GT B and GT C, were manually created to conduct step 1 , step 2 and the entire object-detection method, respectively, by researchers who are familiar with the construction/form of the slab and beam-and-slab bridges (Figure 10). The GT datasets are optimal desired outputs to compare against those generated from the framework. The clean-up time $\left(T_{1}\right)$ and the object-detection time $\left(T_{2}\right)$ were recorded for the manual operation as well as for the framework.

Then, for process 2, a set of 3D geometric models - that is, GT D was manually created to compare against the resulting LOD 250-300 gDTs. Again, the manual refinement time $\left(T_{3}\right)$ and the twinning time $\left(T_{4}\right)$ were recorded for the manual operation as well as for the framework. The manual operation time, as well as the twinning time of the framework, is given in the next section.

\subsection{Experiments}

The experiments of the framework start by determining the two key discriminative parameters $\rho_{1}$ and $\rho_{2}$ in process 1 . They were statistically estimated by comparing against GT A and GT B using point-wise performance metrics precision $\left(P_{\mathrm{r}}\right)$, recall $(R)$ and $F_{1}$ score $\left(F_{1}\right)$. A grid search was conducted over the value space $(0,1)$ and computed the empirical receiver operating characteristic (ROC). The optimal values of $\rho_{1}$ and $\rho_{2}-$ that is, $\rho_{1}^{*}$ and $\rho_{2}^{*}$ - were identified when the distance to the perfect classification in the ROC was minimised. Then, the entire method was evaluated with the other parameters deduced based on $\rho_{1}^{*}$ and $\rho_{2}^{*}$. For each bridge, the evaluation was conducted using both bounding-box-wise and point-wise metrics (Table 3). The average $P_{\mathrm{r}}, R$ and $F_{1}$ of bounding-box-wise component detection for all ten bridges were $100,98.5$ and $99.2 \%$, respectively.

For point-wise evaluation, the micro-average score of $P_{\mathrm{r}} / R / F_{1}$ was $98.4 \%( \pm 3.1 \%)$ for the ten bridges. The false discovery rate (FDR) revealed that some boundary points between adjacent point

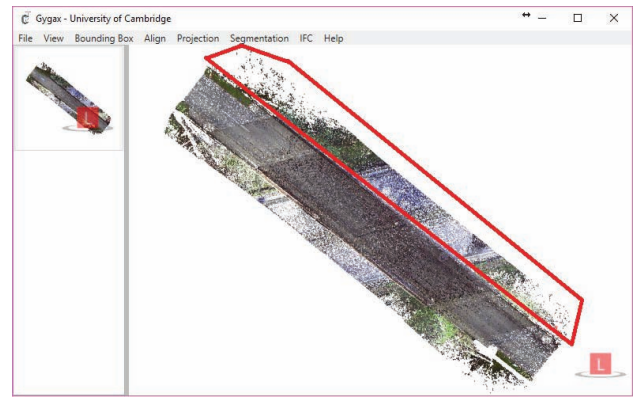

(a)

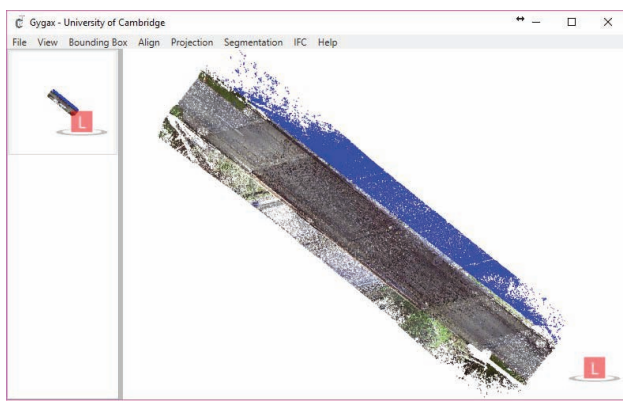

(b)

Figure 9. Top view of bridge 1: (a) clipping polygons; (b) selected points are coloured and removed 
Smart Infrastructure and Construction Volume 172 Issue 1
A benchmarked framework for geometric digital twinning of slab and beam-and-

slab bridges

Lu and Brilakis

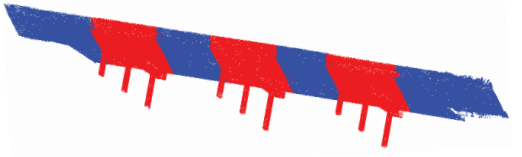

(a)

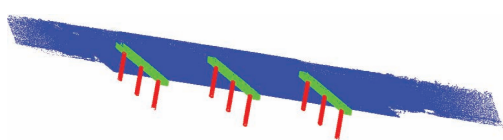

(c)

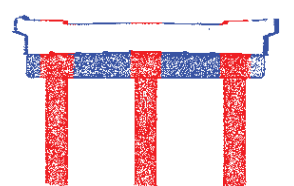

(b)

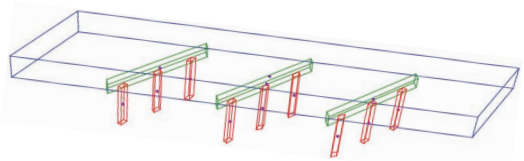

(d)

Figure 10. Process 1: (a) GT A of step 1; (b) GT B of step 2; (c) GT C point clusters of the entire method; (d) GT C oriented bounding boxes of the point clusters (example: bridge 1)

Table 3. Summarised performance of the Lukis framework for EIRs 1, 2, 3 and 6

\begin{tabular}{|c|c|c|c|c|c|c|}
\hline & \multicolumn{3}{|c|}{ Bounding-box-wise: \% } & \multirow{2}{*}{$\frac{\text { Point-wise }(\%)}{\text { Micro-average } \overline{P_{r}} / \bar{R} / \overline{F_{1}}}$} & \multirow{2}{*}{\multicolumn{2}{|c|}{$\overline{C 2 C}: \mathrm{cm}$}} \\
\hline & $\overline{P_{r}}$ & $\bar{R}$ & $\overline{F_{1}}$ & & & \\
\hline EIRs 1 and 3 & 100 & 98.5 & 99.2 & 98.4 & & - \\
\hline EIRs 2 and 6 & & - & & - & $\begin{array}{c}\text { Ten bridges } \\
7.05\end{array}$ & $\begin{array}{l}\text { Eight bridges (without bridges } 7 \text { and 10) } \\
5.6\end{array}$ \\
\hline
\end{tabular}

clusters were detected as false positives (FPs) - for example, bridge $1 \mathrm{FDR}_{\text {pier cap }} 8.6 \%$ and bridge $7 \mathrm{FDR}_{\text {pier }} 2.75 \%$ (detailed results can be found in the paper by $\mathrm{Lu}$ et al. (2019)). Although the number of these FP points was limited, the effectiveness of process 2 would be affected due to the incorrectly generated concave hulls. Thus, the FPs in each point cluster generated from process 1 should be removed before implementing process 2 . To do so, the developed cropping function presented earlier was used (see Figure 9) to select manually the FP points in each point cluster followed by removing them (Figure 11).
The framework then used the refined point clusters as inputs to twin the bridges in IFC format. The twinning quality of both the manually generated models - that is, GT D - and the ones generated from the framework were evaluated and compared using distance-based cloud-to-cloud (C2C) metrics. To do so, GT $\mathrm{D}$ and the automated models were converted from the framework in IFC format into point clouds.

Then, the framework point clouds (denoted 'Lukis') and the GT point clouds (denoted 'GT') of each bridge were compared

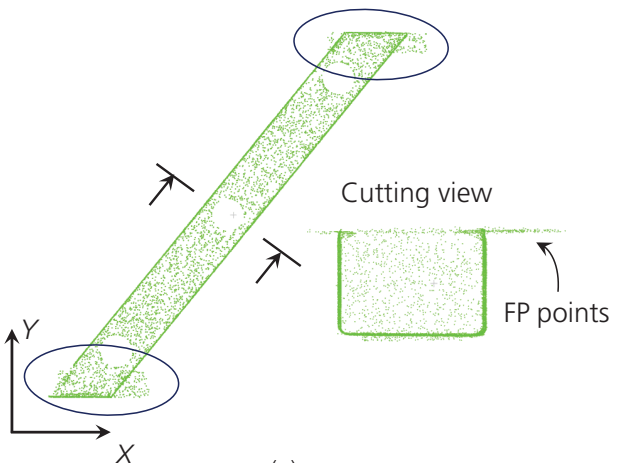

(a)

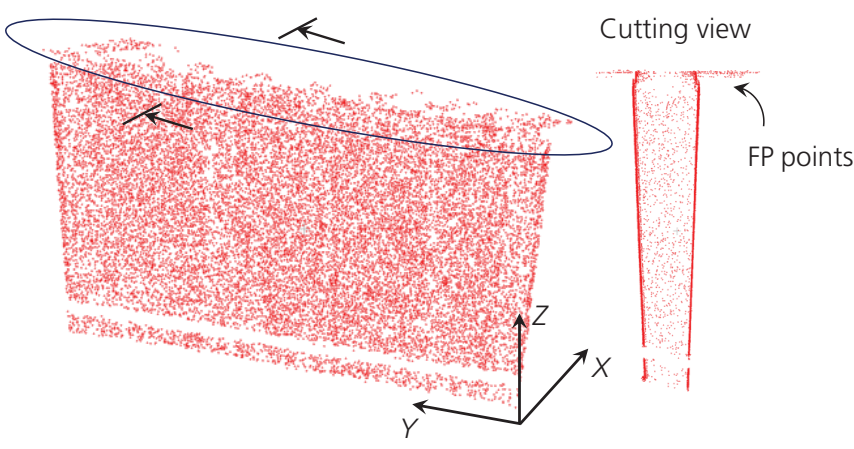

(b)

Figure 11. FP points around boundaries: bridge 1 pier cap 2 (a); bridge 7 pier (b) 
Table 4. Time breakdown of manual operation and the Lukis framework

\begin{tabular}{lcccccc} 
& Clean-up $\bar{T}_{1}: \min$ & Object detection $\bar{T}_{2}: \min$ & Refinement $\bar{T}_{3}: \min$ & Twinning $\bar{T}_{4}: \mathbf{m i n}$ & Overall: $\mathbf{m i n}$ \\
\hline Manual & 58.1 & 12.0 & 9.0 & 1656 & 1735.1 \\
Lukis & 52.0 & 8.3 & 7.8 & 0.63 & 68.7
\end{tabular}

against their original point clouds (denoted 'Real'). In total, six out of ten bridges were modelled better by using Lukis than by the manual operation. There are some challenging scenarios, such as bridge 7 and bridge 10 , where $\mathrm{C} 2 \mathrm{C}$ values were significant,

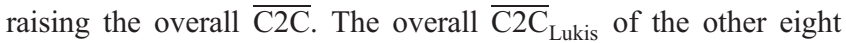
bridge gDTs was $5.6( \pm 1.7) \mathrm{cm}$, while $\overline{\mathrm{C} 2 \mathrm{C}}_{\mathrm{GT}}$ was $7.0( \pm 2.1) \mathrm{cm}$ (detailed results can be found in the paper by $\mathrm{Lu}$ and Brilakis (2019)).

The times spent on data clean-up for both the manual operation and the framework were almost the same (Table 4) - that is $\bar{T}_{1 \text { manual }}=58.1( \pm 6.9) \quad \mathrm{min}$ and $\bar{T}_{1 \text { Lukis }}=52.0( \pm 11.7) \mathrm{min}$. Likewise, the times spent on object detection for both the manual operation and the framework were in the same order of magnitude that is, $\bar{T}_{2 \text { manual }}=12.0( \pm 7.6) \mathrm{min}$ and $\bar{T}_{\text {2Lukis }}=8.3( \pm 0.8) \mathrm{min}$. Then, the times spent on manual refinement $\left(T_{3}\right)$ for the manual operation and for the framework were $9.0( \pm 1.7)$ and $7.8( \pm 1.6) \mathrm{min}$, respectively. The most time-consuming phase in the whole gDT generation is the twinning phase - that is, from point clusters to $3 \mathrm{D}$ shapes. The average time spent was $\bar{T}_{4 \text { manual }}=27.6( \pm 8.2) \mathrm{h}$ ( $\approx 1656 \mathrm{~min}$ ) to twin manually a gDT from labelled point clusters.

The average twinning time of the framework was $37.8( \pm 14.2) \mathrm{s}$, which was trivial compared with the manual operation $\left(\bar{T}_{4 \text { manual }}=27.6 \mathrm{~h}\right)$. Table 4 summarises the time breakdown of the manual operation and that of the framework. As shown, $95.4 \%$ of the overall manual operation time was spent on twinning, whereas this was the most rapid task in the framework (less than $1 \%$ time of the framework). The framework took only a fraction of the manual twinning time $(4 \%)$ to complete the entire workflow. Table 5 shows part of the resulting gDTs of the ten bridges as well as their twinning time $\bar{T}_{4}$.

\section{Conclusions}

This paper presents Lukis, an automated benchmarking framework for generating gDTs for existing highway $\mathrm{RC}$ bridges from point clouds. This work focuses on the compilation of the bridge gDTs while reducing the time and effort required acquiring them. The framework was developed into a software prototype using the coding platform Gygax, which allows practitioners to generate gDTs through a single human-computer interface instead of switching between different software applications using different data formats.

The framework consists of two main processes: process 1 is component detection, aimed at detecting four bridge component types in the form of labelled point clusters, and process 2 is runtime IFC object fitting, aimed at generating the bridge gDT using the four types of point clusters. The framework was implemented on the largest published bridge point-cloud database, consisting of ten highway slab and beam-and-slab RC bridges. In process 1 , the number of misclassified points was limited. This demonstrates the robust performance of the framework on component detection in real-world point clouds featuring defects and how top-down reasoning could facilitate the detection. The limited FP points were present at the boundaries of each point cluster and were suggested to be removed manually in Gygax. These refined labelled point clusters served as the input of process 2 , where the framework successfully generated bridge gDTs, using the four types of point clusters. Distance-based C2C metrics were used to evaluate the twining quality.

The overall $\overline{\mathrm{C} 2 \mathrm{C}}_{\text {Lukis }}$ was $5.6 \mathrm{~cm}$ with a standard deviation of $1.7 \mathrm{~cm}$, if the two non-trivial $\mathrm{C} 2 \mathrm{C}$ values were not counted (i.e. bridge 7 and bridge 10), meaning that an improvement of $20 \%$ was made. Abnormal values exist mainly due to major occlusions rather than twinning deviations. The framework also exhibits difficulties in twinning complex superstructure geometry. Although imperfections exist, experimental results demonstrate that the performance of the framework is consistent and less liable to human error. EIRs 1, 2, 3 and 6 have been largely achieved.

In addition, the framework made an enormous time saving, significantly outperforming the manual practice. The time of clean-up and refinement for the manual process was $67.1 \mathrm{~min}$, while the framework reduced it by $11 \%$. The object-detection and refinement times of the framework were 31 and $13.3 \%$ less than those of the manual operation, respectively. In total, the framework reduced the overall manual twinning time by $96 \%$. Assuming that the framework takes as much time as the manual operation on clean-up, object detection and refinement - that is, $\bar{T}_{1 \text { Lukis }}=\bar{T}_{1 \text { manual }}, \quad \bar{T}_{2 \text { Lukis }}=\bar{T}_{2 \text { manual }}$ and $\bar{T}_{3 \text { Lukis }}=\bar{T}_{3 \text { manual }}-$ then it still can realise a direct time saving of $95.4 \%$. This means that the framework can automate the most time-consuming twinning step, significantly overriding the current practice.

The current version of the framework can twin only the four most important bridge components of the two most representative RC bridge types. In the future, researchers will investigate more bridge types and components with various configurations. The framework has the potential to deal with geometries that are more complex by integrating additional technique layers in each step. Then, it tends to generate undulating-surface and jagged-edge models, which might be over-detailed for end users. Therefore, researchers will investigate how and to what extent to smooth unnecessary undulations and jagged edges.

Next, the approximate alignment tends to generate gaps and overlaps between adjacent slice models of slab. Thus, researchers 
Smart Infrastructure and Construction Volume 172 Issue 1
A benchmarked framework for geometric digital twinning of slab and beam-and-

slab bridges

Lu and Brilakis

Table 5. LOD 250-300 gDTs generated from the framework

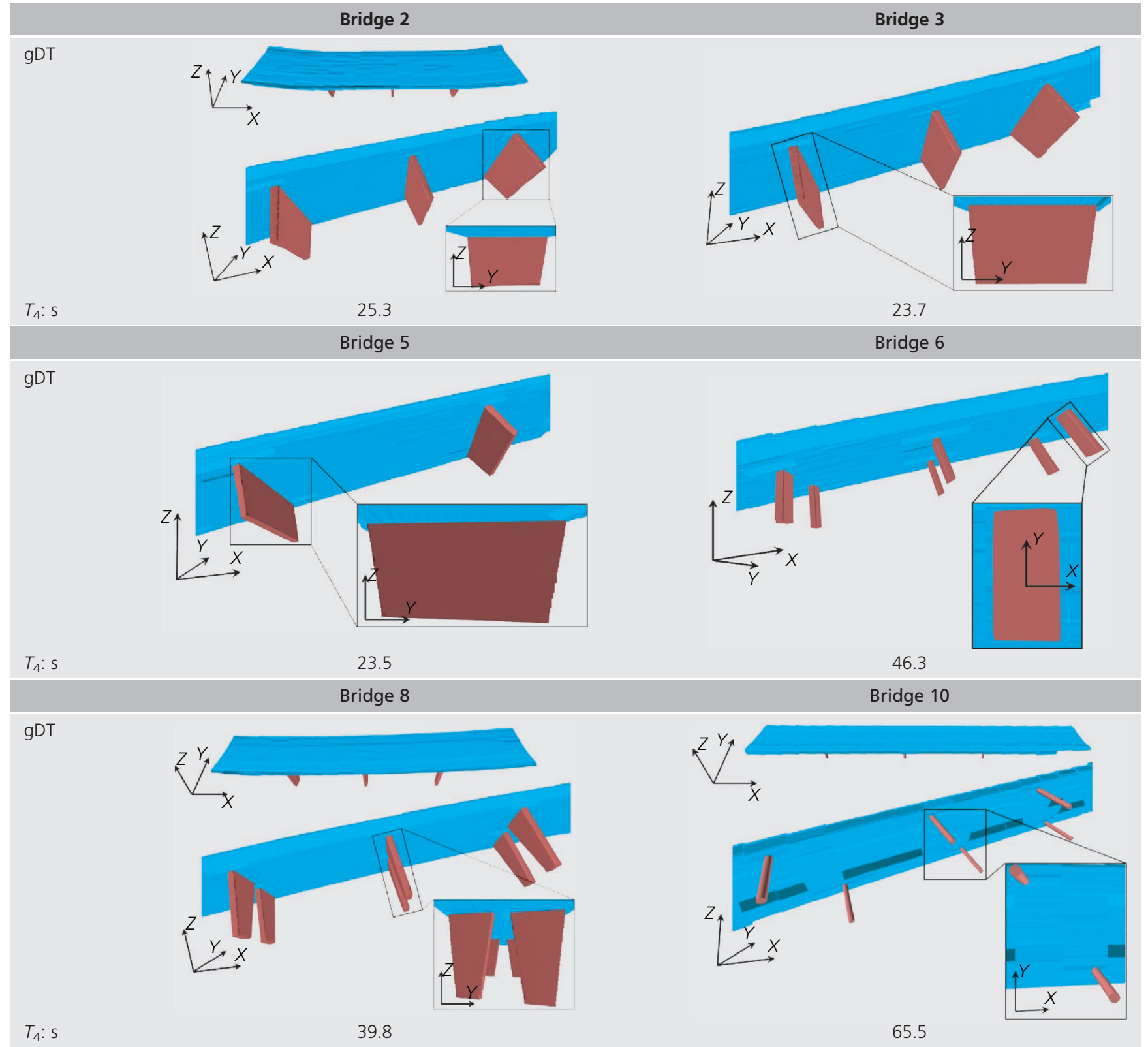

will also investigate a method that can generate a gap-less single curved slab model by sweeping along a $2 \mathrm{D}$ alignment curve using IfcSectionedSolidHorizontal or along an arbitrary 3D curve using IfcFixedReferenceSweptAreaSolid. Last, the framework is not yet fully automatic. Several steps, such as clean-up and refinement, still require human intervention. Approximately $87 \%$ time of the framework was spent on these two manual steps. Future work will focus on automating the remaining manual work, in particular the refinement step, by implementing noise-filtering algorithms.

Overall, the framework, together with other works in this field, is the start of a longer effort to create the toolsets needed to build the national DT roadmap. It lays solid foundations for future research of digital twinning for existing infrastructure.

\section{Acknowledgements}

This research work is supported by the Engineering and Physical Sciences Research Council (EPSRC), Infravation SeeBridge project under grant number 31109806.0007 and Cambridge Trimble Fund. The authors would like to thank them for their support. Any opinions, findings and conclusions or recommendations expressed in this material are those of the authors and do not necessarily reflect the views of EPSRC, Infravation SeeBridge or Trimble. 


\section{REFERENCES}

Adan A and Huber D (2011) 3D reconstruction of interior wall surfaces under occlusion and clutter. Proceedings of the 2011 International Conference on 3D Imaging, Modeling, Processing, Visualization and Transmission (3DIMPVT 2011), Hangzhou, China, pp. 275-281.

Agapaki E, Miatt G and Brilakis I (2018) Prioritising object types for modelling existing industrial facilities. Automation in Construction 96 211-223, https://doi.org/10.1016/j.autcon.2018.09.011.

Ahmed MF, Haas CT and Haas R (2014) Automatic detection of cylindrical objects in built facilities. Journal of Computing in Civil Engineering 28(3): 1-11, https://doi.org/10.1061/(ASCE)CP.1943-5487.0000329.

Armeni I, Sener O, Zamir AR et al. (2016) 3D semantic parsing of largescale indoor spaces. Proceedings of the 2016 IEEE Conference on Computer Vision and Pattern Recognition (CVPR), Las Vegas, NV, USA, pp. 1534-1543.

ASCE (American Society of Civil Engineers) (2013) 2013 Report Card for America's Infrastructure, Bridges. ASCE, Reston, VA, USA. See http://2013.infrastructurereportcard.org/ (03/04/2019).

ASCE (2017) 2017 Report Card for America's Infrastructure, Bridges. ASCE, Reston, VA, USA. See https:/www.infrastructurereportcard. org/cat-item/bridges/ (accessed 03/04/2019).

Ashworth S, Tucker M and Druhmann C (2017) Employer's Information Requirements (EIR): a BIM case study to meet client and facility manager needs. In Research Papers for EUROFM'S 16th Research Symposium, EFMC 2017 (Nielsen SB, Jensen PA and Brin R (eds)). Polyteknisk Boghandel og Forlag, Lyngby, Denmark, pp. 112-121.

Ashworth S, Tucker M and Druhmann CK (2019) Critical success factors for facility management employer's information requirements (EIR) for BIM. Facilities 37(1/2): 103-111, https://doi.org/10.1108/F-022018-0027.

Belsky M, Eastman C, Sacks R et al. (2014) Interoperability for precast concrete building models. PCI Journal 59(2): 144-155, https://doi.org/ 10.15554/pcij.03012014.144.155.

Borrmann A and Berkhahn V (2018) Principles of geometric modeling. In Building Information Modeling (Borrmann A, König M, Koch C and Beetz J (eds)). Springer, Cham, Switzerland, pp. 27-41.

Borrmann A, König M, Koch C and Beetz J (2018a) Building Information Modeling: Why? What? How? In Building Information Modeling (Borrmann A, König M, Koch C and Beetz J (eds)). Springer, Cham, Switzerland, pp. 1-24.

Borrmann A, Beetz J, Koch C, Liebich T and Muhic S (2018b) Industry Foundation Classes: a standardised data model for the vendor-neutral exchange of digital building models. In Building Information Modeling (Borrmann A, König M, Koch C and Beetz J (eds)). Springer, Cham, Switzerland, pp. 81-126.

Buckley B and Logan K (2017) The Business Value of BIM for Infrastructure 2017. Dodge Data \& Analytics, Hamilton, NJ, USA. See https://www2.deloitte.com/content/dam/Deloitte/us/Documents/ finance/us-fas-bim-infrastructure.pdf (accessed 03/04/2019).

ClearEdge3D (2019) Structure Modelling Tools. ClearEdge3D, Manassas, VA, USA. See https://www.clearedge3d.com/ (accessed 16/06/2019).

Davila Delgado JM, Butler LJ, Gibbons N et al. (2017) Management of structural monitoring data of bridges using BIM. Proceedings of the Institution of Civil Engineers - Bridge Engineering 170(3): 204-218, https://doi.org/10.1680/jbren.16.00013.

Díaz-Vilariño L, Khoshelham K, Martínez-Sánchez J and Arias P (2015) 3D modeling of building indoor spaces and closed doors from imagery and point clouds. Sensors 15(2): 3491-3512, https://doi.org/10.3390/ s150203491.

Dimitrov A and Golparvar-Fard M (2015) Segmentation of building point cloud models including detailed architectural/structural features and MEP systems. Automation in Construction 51(C): 32-45, https://doi. org/10.1016/j.autcon.2014.12.015.

Dimitrov A, Gu R and Golparvar-Fard M (2016) Non-uniform B-spline surface fitting from unordered $3 \mathrm{D}$ point clouds for as-built modeling.
Computer-aided Civil and Infrastructure Engineering 31(7): 483-498, https://doi.org/10.1111/mice.12192.

Dore C and Murphy M (2014) Semi-automatic generation of as-built BIM façade geometry from laser and image data. Journal of Information Technology in Construction 19: 20-46.

FHWA (Federal Highway Administration) (2012) Estimated 2012 Costs to Replace or Rehabilitate Structurally Deficient Bridges. FHWA, Washington, DC, USA. See https://www.fhwa.dot.gov/bridge/nbi/ sd2012.cfm (accessed 03/04/2019).

Fink T (2018) BIM for structural engineering. In Building Information Modeling (Borrmann A, König M, Koch C and Beetz J (eds)). Springer, Cham, Switzerland, pp. 329-336.

Flaig KD and Lark RJ (2000) The development of UK bridge management systems. Proceedings of the Institution of Civil Engineers - Transport 141(2): 99-106, https://doi.org/10.1680/tran.2000.141.2.99.

Grieves M and Vickers J (2016) Digital twin: mitigating unpredictable, undesirable emergent behavior in complex systems. In Transdisciplinary Perspectives on Complex Systems: New Findings and Approaches (Kahlen FJ, Flumerfelt S and Alves A (eds)). Springer, Cham, Switzerland, pp. 85-113.

Hafeez MA, Ahmad AM, Chahrour R et al. (2016) Principles and recommendations for client information requirements for BIM enabled construction projects in Qatar. International Journal of Product Lifecycle Management 9(3): 198-218, https://doi.org/10.1504/ijplm. 2016.10001531.

Hüthwohl P, Armeni I, Fathi H and Brilakis I (2017) Gygax Construction IT Research Platform for $2 D \& 3 D$. GitHub, San Francisco, CA, USA. See https://github.com/ph463/Gygax (accessed 18/04/2020).

Hüthwohl P, Brilakis I, Borrmann A and Sacks R (2018) Integrating RC bridge defect information into BIM models. Journal of Computing in Civil Engineering 32(3): article 04018013, https://doi.org/10.1061/ (ASCE)CP.1943-5487.0000744.

ISO (International Organization for Standardization) (2017) ISO/DIS 19650-1: Organization of information about construction works information management using building information modelling - Part 1: concepts and Principles. ISO, Geneva, Switzerland.

Jung J, Hong S, Jeong S et al. (2014) Productive modeling for development of as-built BIM of existing indoor structures. Automation in Construction 42: 68-77, https://doi.org/10.1016/j.autcon.2014.02. 021

Kedar A (2014) Guide for Documenting Bridges and Road Structures Data. Kedmor Engineers Ltd, Givataim, Israel.

Kedar A (2016) SeeBridge Project Document: Criteria for Evaluation of Complete SeeBridge System.

Kern WF and Bland JR (1948) Spherical segment. In Solid Mensuration with Proofs, 2nd edn. Wiley New York, NY, USA, pp. 97-102.

Koch C and König M (2018) Data modeling. In Building Information Modeling (Borrmann A, König M, Koch C and Beetz J (eds)). Springer, Cham, Switzerland, pp. 43-62.

Koppula HS, Anand A, Joachims T and Saxena A (2011) Semantic labeling of 3D point clouds for indoor scenes. Advances in Neural Information Processing Systems 24: 25th Annual Conference on Neural Information Processing Systems 2011, NIPS 2011.

Laefer DF and Truong-Hong L (2017) Toward automatic generation of 3D steel structures for building information modelling. Automation in Construction 74: 66-77, https://doi.org/10.1016/j.autcon.2016.11.011.

Lu R and Brilakis I (2019) Digital twinning of existing reinforced concrete bridges from labelled point clusters. Automation in Construction 105: article 102837, https://doi.org/10.1016/j.autcon.2019.102837.

Lu R, Brilakis I and Middleton CR (2018) Detection of Structural Components in Point Clouds of Existing RC Bridges. Zenodo, European Organization for Nuclear Research, Geneva, Switzerland. See http://doi.org/10.5281/zenodo.1233844 (accessed 18/04/2020).

Lu R, Brilakis I and Middleton CR (2019) Detection of structural components in point clouds of existing RC bridges. Computer-aided 
Civil and Infrastructure Engineering 34(3): 191-212, https://doi.org/ 10.1111/mice.12407.

Ma L, Sacks R, Kattel U and Bloch T (2018) 3D object classification using geometric features and pairwise relationships. Computer-aided Civil and Infrastructure Engineering 33(2): 152-164, https://doi.org/10. $1111 /$ mice. 12336 .

Macher H, Landes T and Grussenmeyer P (2017) From point clouds to building information models: $3 \mathrm{D}$ semi-automatic reconstruction of indoors of existing buildings. Applied Sciences 7(10): article 1030, https://doi.org/10.3390/app7101030

Marton ZC, Rusu RB and Beetz M (2009) On fast surface reconstruction methods for large and noisy point clouds. Proceedings of the 2009 IEEE International Conference on Robotics and Automation Kobe, Japan, pp. 3218-3223.

Maturana D and Scherer S (2015) VoxNet: a 3D Convolutional Neural Network for real-time object recognition. Proceedings of the 2015 IEEE/RSJ International Conference on Intelligent Robots and Systems (IROS), Hamburg, Germany, pp. 922-928.

NAO (National Audit Office) (2018) A Short Guide to the Department for Transport. NAO, London, UK. See https://www.nao.org.uk/wpcontent/uploads/2018/02/A-Short-Guide-to-the-Department-forTransport-2017.pdf (accessed 18/04/2020).

Ochmann S, Vock R, Wessel R and Klein R (2016) Automatic reconstruction of parametric building models from indoor point clouds. Computers \& Graphics 54: 94-103, https://doi.org/10.1016/j.cag.2015.07.008.

Oesau S, Lafarge F and Alliez P (2014) Indoor scene reconstruction using feature sensitive primitive extraction and graph-cut. ISPRS Journal of Photogrammetry and Remote Sensing 90: 68-82, https://doi.org/10. 1016/j.isprsjprs.2014.02.004.

Parrott A and Lane W (2017) Industry 4.0 and the Digital Twin. Deloitte University Press, New York, NY, USA. See https://www2.deloitte. com/insights/us/en/focus/industry-4-0/digital-twin-technology-smartfactory.html (accessed 14/10/2019).

Patil AK, Holi P, Lee SK and Chai YH (2017) An adaptive approach for the reconstruction and modeling of as-built $3 \mathrm{D}$ pipelines from point clouds. Automation in Construction 75: 65-78, https://doi.org/10.1016/ j.autcon.2016.12.002

Perez-Gallardo Y, Cuadrado JLL, Crespo ÁG and de Jesús CG (2017) GEODIM: a semantic model-based system for 3D recognition of industrial scenes. In Intelligent Systems Reference Library. In Current Trends on Knowledge-based Systems (Alor-Hernández G and Valencia-García R (eds)). Springer, Cham, Switzerland, pp. 137-159.

Qi CR, Su H, Mo K and Guibas LJ (2017) PointNet: deep learning on point sets for 3D classification and segmentation. Proceedings - 30th IEEE Conference on Computer Vision and Pattern Recognition, CVPR 2017.

Rabbani TR (2006) Automatic Reconstruction of Industrial Installations Using Point Clouds and Images. Netherlands Geodetic Commission, Delft, the Netherlands. Publications on Geodesy, 62. See http:// resolver.tudelft.nl/uuid:0012068e-93b4-4bd9-a9b3-9c579ae7c91a (accessed 03/04/2019).

Riveiro B, DeJong MJ and Conde B (2016) Automated processing of large point clouds for structural health monitoring of masonry arch bridges. Automation in Construction 72(3): 258-268, https://doi.org/10.1016/j. autcon.2016.02.009.

Sacks R, Ma L, Yosef R et al. (2017) Semantic enrichment for building information modeling: procedure for compiling inference rules and operators for complex geometry. Journal of Computing in Civil Engineering 31(6), https://doi.org/10.1061/(ASCE)CP.1943-5487. 0000705 .

Sacks R, Eastman C, Lee G and Teicholz P (2018a) BIM Handbook. BIM Handbook, https://doi.org/10.1002/9781119287568.

Sacks R, Kedar A, Borrmann A et al. (2018b) SeeBridge as next generation bridge inspection: Overview, Information Delivery Manual and Model View Definition. Automation in Construction 90: 134-145, https://doi. org/10.1016/j.autcon.2018.02.033
Schnabel R, Wahl R and Klein R (2007) Efficient RANSAC for pointcloud shape detection. Computer Graphics Forum 26(2): 214-226, https://doi.org/10.1111/j.1467-8659.2007.01016.x.

Son H, Kim C and Kim C (2013) Knowledge-based approach for 3D reconstruction of as-built industrial plant models from laser-scan data. Proceedings of the 30th International Symposium on Automation and Robotics in Construction and Mining (ISARC 2013), Montréal, QC, Canada, pp. 885-893.

Son H, Kim C and Kim C (2015) 3D reconstruction of as-built industrial instrumentation models from laser-scan data and a 3D CAD database based on prior knowledge. Automation in Construction 49(Part B): 193-200, https://doi.org/10.1016/j.autcon. 2014.08.007.

Su Y, Bethel J and Hu S (2016) Octree-based segmentation for terrestrial LiDAR point cloud data in industrial applications. ISPRS Journal of Photogrammetry and Remote Sensing 113: 59-74, https://doi.org/10. 1016/j.isprsjprs.2016.01.001.

Teizer J and Melzner J (2018) BIM for construction safety and health. In Building Information Modeling (Borrmann A, König M, Koch C and Beetz J (eds)). Springer, Cham, Switzerland, pp. 349-365.

Treeck C van, Wimmer R and Maile T (2018) BIM for energy analysis. In Building Information Modeling (Borrmann A, König M, Koch C and Beetz J (eds)). Springer, Cham, Switzerland, pp. 337-347.

Truong-Hong L, Laefer DF, Hinks T and Carr H (2013) Combining an angle criterion with voxelization and the flying voxel method in reconstructing building models from LiDAR data. Computer-aided Civil and Infrastr ucture Engineering 28(2): 112-129, https://doi.org/ 10.1111/j.1467-8667.2012.00761.x.

Valero E, Adán A and Cerrada C (2012) Automatic method for building indoor boundary models from dense point clouds collected by laser scanners. Sensors 12(12): 16099-16115, https://doi.org/10.3390/ s121216099.

Valero E, Adán A and Bosché F (2016) Semantic 3D reconstruction of furnished interiors using laser scanning and RFID technology. Journal of Computing in Civil Engineering 30(4): article 04015053, https://doi org/10.1061/(ASCE)CP.1943-5487.0000525.

Venugopal M, Eastman CM, Sacks R and Teizer J (2012) Semantics of model views for information exchanges using the industry foundation class schema. Advanced Engineering Informatics 26(2): 411-428, https://doi.org/10.1016/j.aei.2012.01.005.

Vo AV, Truong-Hong L, Laefer DF and Bertolotto M (2015) Octree-based region growing for point cloud segmentation. ISPRS Journal of Photogrammetry and Remote Sensing 104: 88-100, https://doi.org/10. 1016/j.isprsjprs.2015.01.011.

Walsh SB, Borello DJ, Guldur B and Hajjar JF (2013) Data processing of point clouds for object detection for structural engineering applications. Computer-aided Civil and Infrastructure Engineering 28(7): 495-508, https://doi.org/10.1111/mice.12016.

Wang C, Cho YK and Kim C (2015) Automatic BIM component extraction from point clouds of existing buildings for sustainability applications. Automation in Construction 56: 1-13, https://doi.org/10.1016/j.autcon. 2015.04.001.

Webb GT (2014) Structural Health Monitoring of Bridges. PhD thesis, University of Cambridge, Cambridge, UK.

Xiao J and Furukawa Y (2014) Reconstructing the world's museums. International Journal of Computer Vision 110(3): 243-258, https://doi. org/10.1007/s11263-014-0711-y.

Xiong X, Adan A, Akinci B and Huber D (2013) Automatic creation of semantically rich $3 \mathrm{D}$ building models from laser scanner data. Automation in Construction 31: 325-337, https://doi.org/10.1016/j. autcon.2012.10.006.

Xu Y, Tuttas S, Hoegner L and Stilla U (2018) Voxel-based segmentation of $3 \mathrm{D}$ point clouds from construction sites using a probabilistic connectivity model. Pattern Recognition Letters 102: 67-74, https:// doi.org/10.1016/j.patrec.2017.12.016. 
Smart Infrastructure and Construction

Volume 172 Issue 1
A benchmarked framework for geometric

digital twinning of slab and beam-and-

slab bridges

Lu and Brilakis
Zhang G, Vela PA and Brilakis I (2014) Automatic generation of as-built geometric civil infrastructure models from point cloud data. In Computing in Civil and Building Engineering (2014) (Issa RR and Flood I (eds)). ASCE, Reston, VA, USA, pp. 406-413.
Zhang G, Vela PA, Karasev P and Brilakis I (2015) A sparsity-inducing optimization-based algorithm for planar patches extraction from noisy point-cloud data. Computer-aided Civil and Infrastructure Engineering 30(2): 85-102, https://doi.org/10.1111/mice.12063.

\section{How can you contribute?}

To discuss this paper, please email up to 500 words to the editor at journals@ice.org.uk. Your contribution will be forwarded to the author(s) for a reply and, if considered appropriate by the editorial board, it will be published as discussion in a future issue of the journal.

Proceedings journals rely entirely on contributions from the civil engineering profession (and allied disciplines). Information about how to submit your paper online is available at www.icevirtuallibrary.com/page/authors, where you will also find detailed author guidelines. 